\title{
Perturbación post-huracán Dean en el hábitat y la abundancia relativa de vertebrados mayores de la Selva Maya, Quintana Roo, México
}

\author{
Post-hurricane Dean impact on habitat and its influence on the relative abundance of large \\ vertebrates in the Selva Maya, Quintana Roo, Mexico
}

\author{
Pablo Jesús Ramírez-Barajas ${ }^{1 \otimes}$, Gerald A. Islebe ${ }^{2}$ y Nuria Torrescano-Valle ${ }^{2}$ \\ ${ }^{I}$ Red Biología y conservación de vertebrados, Instituto de Ecología, A. C. Carretera Antigua a Coatepec 351, El Haya, 91070 Xalapa, Veracruz, México. \\ ${ }^{2}$ El Colegio de la Frontera Sur. Avenida Centenario Km. 5.5, 77014 Chetumal, Quintana Roo, México. \\ 凶ab_rb@yahoo.com.mx
}

\begin{abstract}
Resumen. Se evaluó la intensidad de daño en el hábitat después del huracán Dean y su influencia en la abundancia de fauna, en la selva del centro sur del estado de Quintana Roo, México. Se situaron 20 sitios de muestreo ordenados de mayor a menor afectación. En cada sitio se realizó un muestreo de vegetación para determinar las características del hábitat y el impacto de huracán. Para la fauna, cada sitio fue muestreado para obtener índices de abundancia relativa de rastros (rastros $/ \mathrm{km}$ ). Se realizaron entrevistas con informantes clave para determinar las especies arbóreas consumidas por la fauna. El porcentaje de daño disminuyó gradualmente hasta menos de $4 \%$ a una distancia de 120 $\mathrm{km}$ perpendiculares al huracán. El hábitat fue relativamente homogéneo y no se encontraron diferencias entre los parámetros y atributos medidos en la vegetación, la única variable que resultó diferente fue el porcentaje de daño en los árboles. El 65\% de las especies arbóreas, fueron registradas como alimento para frugívoros, herbívoros y omnívoros. En sitios de alto impacto, la abundancia de herbívoros fue menor mientras que la abundancia de omnívoros fue mayor. El daño en los árboles fue la única variable que se correlacionó negativamente con la abundancia de los herbívoros, lo cual no fue evidente con los omnívoros.
\end{abstract}

Palabras clave: disturbio natural, grupos funcionales, herbívoros, omnívoros, selva mediana subperennifolia, sureste de México.

\begin{abstract}
We assessed the damage on the habitat after Hurricane Dean and its influence on the abundance of wildlife. We used 20 sampling sites along an area of high to low impact. At each site, vegetation sampling was conducted to determine the characteristics of habitat and the impact of hurricane Dean. For fauna, each site was sampled to obtain indices of relative tracks abundance (tracks $/ \mathrm{km}$ ). Interviews were conducted with key informants to determine which tree species were consumed by the local fauna. Damage gradually declined to less than $4 \%$ at a distance of 120 $\mathrm{km}$ perpendicular to the hurricane. The habitat was relatively homogeneous and no differences were found between the parameters and attributes measured in the vegetation, the only variable that differed was the percentage of trees damage. Of tree species, $65 \%$ were reported as food for frugivorous, herbivorous and omnivorous species. At highimpact sites the abundance of herbivores was lower while abundance of omnivores was higher. Damage to trees species was the only variable that negatively correlated with the abundance of herbivorous species, which was not evident with omnivorous species.
\end{abstract}

Key words: functional groups, herbivorous, omnivorous, natural disturbance, tropical medium-statured forest, Southeast Mexico.

\section{Introducción}

La pérdida, fragmentación y degradación de hábitat son los 3 principales procesos que contribuyen a los cambios en el paisaje y suelen ser las mayores amenazas para la biodiversidad (Fischer y Lindenmayer, 2007). Aunque los 3 procesos pueden presentarse simultáneamente, la degradación puede suceder independientemente dentro de un paisaje continuo (Fischer y Lindenmayer, 2007; Mortelliti

Recibido: 11 octubre 2011; aceptado: 21 mayo 2012 et al., 2010). El proceso de degradación implica el deterioro gradual de la calidad de hábitat (Mortelliti et al., 2010) y en el caso de disturbios naturales como los huracanes, el hábitat experimenta una degradación temporal y espacial. Se entiende por disturbio cualquier evento repentino en el tiempo que altera la estructura de los ecosistemas, comunidades y poblaciones, y que cambia la disponibilidad de los recursos, sustrato o medio físico, mientras que una perturbación es el cambio de un estado, conducta o trayectoria de un sistema ecológico (Pickett y White, 1985). En este sentido, un disturbio es el evento o agente de cambio (e.g., 
huracanes, incendios) y una perturbación es la condición dejada por este evento.

Los huracanes se consideran los eventos de perturbación natural más comunes para los ecosistemas del Caribe (Tanner et al., 1991); por lo cual, forman parte de la dinámica natural del clima y contribuyen a la reorganización de la estructura de la comunidad, así como a los procesos ecosistémicos. Estos fenómenos permiten comprender diversos procesos ecológicos, ya que representan verdaderos laboratorios naturales donde excepcionalmente se pueden observar y medir las reacciones y procesos de los ecosistemas con un punto de partida bien definido en espacio y tiempo. A pesar de esto, es relativamente reciente la documentación sobre los efectos en los ecosistemas, poblaciones y especies (Zimmerman et al., 1996).

En un hábitat degradado, una especie puede declinar, permanecer con baja abundancia o ser incapaz de reproducirse (Felton et al., 2003; Hazell et al., 2004). Por lo cual, el monitoreo de especies de vertebrados mediante su presencia-ausencia o abundancia y densidad aporta información relevante sobre el estado de conservación de las poblaciones y su hábitat (Chiarello, 2000). Sin embargo, a pesar de que la abundancia de fauna puede ser un indicador de la calidad de hábitat, también puede ser respuesta a diversos factores tanto climáticos, estacionales y espaciales, así como a interacciones sociales, capacidad reproductiva y características ecológicas de las especies (van Horne, 1983; Kreuzer y Huntly, 2003). Por lo tanto, es necesario complementar las evaluaciones de fauna con evaluaciones del hábitat y las condiciones paisajísticas del área para tener una interpretación más completa del complejo ecosistema-especies.

Cabe destacar que el efecto de perturbaciones naturales en el hábitat y las consecuencias en la fauna, en general, ha sido escasamente abordado, a pesar de la íntima relación del hábitat como herramienta potencial de interpretación de las abundancias (Worman y Chapman, 2006). Mientras que una gran proporción de los vertebrados tropicales son frugívoros, de manera recíproca, gran variedad de especies arbóreas producen frutos y atraen a estos frugívoros como una estrategia para la dispersión de semillas (Fleming et al., 1987; Chapman et al., 1994; Milton, 2008). Por lo tanto, el hábitat puede ser evaluado en función de la disponibilidad de alimento para la fauna y de las condiciones de conservación de la vegetación predominante en el área de estudio. La composición numérica de este amplio gremio de consumidores primarios pone en relieve la importancia de investigar su ecología y la evaluación del hábitat. De esta forma, el efecto de perturbaciones naturales en los ecosistemas representa una oportunidad para medir los diferentes patrones de respuesta tanto del hábitat como de la fauna residente.
El presente trabajo evaluó la calidad de hábitat en función de los atributos ecológicos de la vegetación y el disturbio del huracán Dean, de acuerdo con la intensidad del daño, así como la influencia de estas variables de hábitat en la abundancia de 9 especies de vertebrados de talla mediana y grande. Se espera que a mayor daño en la vegetación se tenga una menor calidad de hábitat, así como un efecto negativo en la abundancia relativa de vertebrados de acuerdo con sus requerimientos ecológicos.

\section{Materiales y métodos}

Área de estudio. El área de estudio se localiza en las zonas de influencia oeste de la Reserva de la Biosfera Sian Ka'an y en los ejidos forestales del centro-sur del estado de Quintana Roo entre las coordenadas $19^{\circ} 07^{\prime} 55^{\prime \prime} \mathrm{N}, 88^{\circ} 01^{\prime} 03^{\prime \prime}$ O y $19^{\circ} 13^{\prime} 23^{\prime \prime} \mathrm{N}, 8^{\circ} 24^{\prime} 18^{\prime \prime} \mathrm{O}$. El clima es templado subhúmedo, con temperatura media anual de $26^{\circ} \mathrm{C} \mathrm{y}$ precipitación promedio de $1200 \mathrm{~mm}$ anuales, con lluvias máximas entre junio y septiembre (Orellana et al., 2009). A esta región se le han asignado altos valores de integridad ecológica funcional y alta importancia en su función en el Corredor Biológico Mesoamericano que enlaza las reservas de la biosfera Sian Ka'an y Calakmul (Arriaga et al., 2000; Fig. 1).

El huracán Dean recorrió de este a oeste la península de Yucatán. Arribó cerca del poblado de Mahahual con la máxima categoría (escala 5 Saffir-Simpson) el 21 de agosto de 2007, con vientos de hasta $280 \mathrm{~km} / \mathrm{h}$ y una precipitación de $81 \mathrm{~mm}$ (CONAGUA, 2007). Al momento del impacto, este fenómeno estuvo acompañado de precipitación particularmente baja, en comparación con el huracán Gilberto (1988) que presentó una precipitación de $350 \mathrm{~mm}$ (CONAGUA, 2007; NOAA, 2011), por lo que su capacidad de afectación radicó principalmente en la fuerza de sus vientos.

Se situaron 20 sitios de muestreo en toda el área de estudio, el arreglo espacial de los sitios se realizó considerando la afectación a partir de $35 \mathrm{~km}$ hasta $120 \mathrm{~km}$ perpendicularmente a la ruta del ojo del huracán (Figs. 1, 2), de tal forma que correspondieran con un mayor a menor grado de afectación del hábitat. Cada sitio consistió de 3 transectos de $3 \mathrm{~km}$ de longitud (9 $\mathrm{km}$ por sitio). La disposición espacial de los mismos dentro de cada sitio permitió que cada transecto fuera independiente de los otros. En cada sitio, la separación entre transectos fue al menos de $700 \mathrm{~m}$, mientras que los sitios estuvieron separados más de $2 \mathrm{~km}$, bajo un esquema que permitiera independencia espacial entre sitios (Fig. 1).

Muestreo de fauna. Las especies focales para el registro de rastros en este estudio fueron 9 vertebrados de talla mediana $(2.5-10 \mathrm{~kg})$ y grande $(>10 \mathrm{~kg})$. Una alternativa 


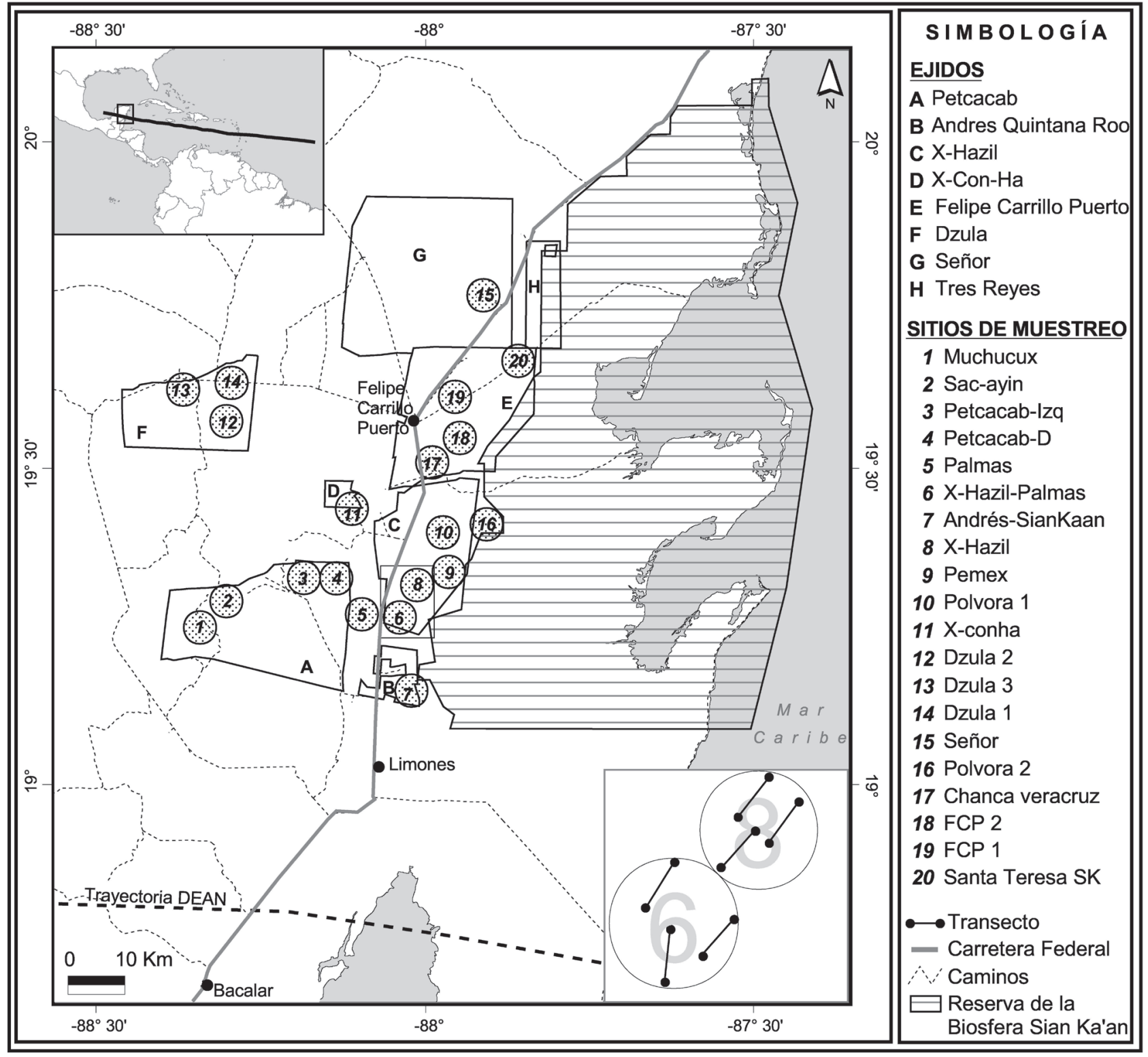

Figura 1. Arreglo espacial de los sitios de muestreo tanto de vegetación como de fauna. El recuadro inferior derecho muestra la disposición de los transectos.

en el caso de baja detectabilidad de fauna en las regiones tropicales es el muestreo de rastros como un método no invasivo (Carrillo et al., 2000; Silveira et al., 2003). Para analizar cuáles características hacen a las poblaciones de estos vertebrados más o menos sensibles al daño por el huracán y a los parámetros y atributos del hábitat, se asignaron grupos funcionales basados en la literatura sobre su dieta (desde los frugívoros estrictos hasta el más omnívoro), de acuerdo con Ramírez-Barajas et al. (2012). Estos grupos incluyen a frugívoros como el cereque (Dasyprocta punctata) y tepezcuintle (Cuniculus paca); a frugívoro- ramoneadores como el venado cola blanca (Odocoileus virginianus) y el temazate (Mazama americana/Mazama pandora); a los frugívoro-omnívoros como el hocofaisán (Crax rubra) y pavo ocelado (Meleagris ocellata); a los omnívoro-frugívoros como el coatí (Nasua narica) y pecarí de collar (Pecari tajacu); y al omnívoro menos dependiente de las plantas como alimento, el armadillo (Dasypus novemcinctus). Debido a que estas especies son principalmente de hábitos terrestres, es posible encontrar indicios de sus actividades (e.g., desplazamientos, refugios), los cuales permiten un registro sistemático a través 
de huellas y señales de actividad sobre el sustrato. La identificación de huellas y rastros se realizó con la ayuda de expertos de campo y guías ilustradas de campo (Aranda, 2000; Murie y Elbroch, 2005; Urquiza-Haas et al., 2011).

La abundancia relativa por especie y por grupo funcional se obtuvo con el índice de abundancia relativa de rastros (IAR), el cual deriva del número de rastros de cada especie por kilómetro caminado (\# rastros $/ \mathrm{km}$ ). Este índice permite realizar comparaciones de abundancias relativas bajo las diferentes circunstancias espaciales y para diferentes grupos de fauna, desde ungulados, roedores mayores y otras especies de vertebrados capaces de dejar huella (Carrillo et al., 2000; Wilson y Delahay, 2001; Krebs, 2006).

El muestreo de rastros es ampliamente utilizado por su fácil ejecución y versatilidad, su bajo costo económico y no requiere la detección de individuos en el momento preciso del muestreo (Wilson y Delahay, 2001; Lyra-Jorge et al., 2008; Roberts, 2011). Aunque este método puede emplearse para el registro de presencia-ausencia de especies (detección- no detección) en condiciones restringidas de presupuesto y tiempo, en el presente estudio se usó para determinar la abundancia relativa de rastros (expresado como un índice de abundancia relativa) por grupos funcionales de las especies focales.

El muestreo de rastros se inició en abril de 2008 (8 meses después del paso del huracán Dean) y finalizó en marzo de 2010 (31 meses después del huracán). Para incluir la variación anual, los muestreos tuvieron lugar durante la estación seca y lluviosa de cada año. El muestreo de rastros se realizó mediante caminatas diurnas (0600-1000 h) a una velocidad promedio de $1 \mathrm{~km} / \mathrm{h}$ (Burnham et al., 1980). Los transectos se marcaron cada 50-100 m para controlar la velocidad y obtener posiciones precisas de los rastros. Los datos de rastros fueron recolectados a lo largo de los transectos lineales. Todos los transectos se localizaron en selva mediana subperennifolia, debido a que es la vegetación predominante y presenta las mejores condiciones de substrato (suelo y hojarasca) para la identificación de rastros, lo cual permite la observación de actividad de la fauna en cualquier periodo del año (Ramírez-Barajas et al., 2012). Durante el periodo post-huracán, cada sitio fue muestreado 7 veces, lo cual hizo un total de $1260 \mathrm{~km}$ en toda el área de estudio.

Muestreo de vegetación. El periodo de muestreo de vegetación se realizó entre 15 y 21 meses post-huracán, desde noviembre de 2008 hasta mayo de 2009 (la estación seca del año). Con el fin de caracterizar las condiciones de la vegetación en el área de estudio, se utilizó el protocolo de muestreo de vegetación de Gentry (1982). Se eligió al azar un transecto en cada uno de los 20 sitios de muestreo. A lo largo de cada transecto se establecieron 10 parcelas de
$50 \times 2 \mathrm{~m}$, separadas cada $50 \mathrm{~m}$ y alternadas a cada lado del transecto, lo cual cubre un total $1000 \mathrm{~m}^{2}$ por sitio (20 $000 \mathrm{~m}^{2}$ en toda el área de estudio). Se realizaron algunas modificaciones al método para la evaluación de los parámetros y atributos del hábitat, cuantificación de árboles de importancia alimenticia para la fauna y evaluación de daños provocados por el huracán (Sánchez e Islebe, 1999; Arroyo-Rodríguez y Mandujano, 2006; Arroyo-Rodríguez et al., 2007).

Se registraron todos los árboles con diámetro mayor de $5 \mathrm{~cm}$ a la altura del pecho (DAP, $1.3 \mathrm{~m}$ ), medida de uso generalizado, de alta correlación con la disponibilidad y abundancia de frutos y estado de madurez de los árboles (Chapman et al., 1992). Además, se anotó el nombre común, la especie, diámetro de copa (cobertura), altura, fenofases (floración-fructificación) y tipo de daño. Se obtuvo el área basal (mediante DAP y altura), la cobertura (con el diámetro de la copa), la densidad total (individuos/ ha) y la riqueza de especies para cada sitio en toda el área de estudio. Estos atributos fueron seleccionados para tener una valoración de las características del hábitat (ArroyoRodríguez y Mandujano, 2006; Arroyo-Rodríguez et al., 2007). Se obtuvo también el índice de valor de importancia de cada especie arbórea (IVI), el cual es un buen descriptor ecológico de las especies. Este índice es adimensional y se estima con la suma de la densidad relativa (árboles $/ \mathrm{m}^{2}$ ), frecuencia (número de veces donde aparece la especie en cada sitio o parcela) y dominancia (área basal total de cada especie; Kent y Coker, 1994).

La cuantificación y descripción de los daños provocados por el huracán Dean en los árboles se realizó, de acuerdo con Sánchez e Islebe (1999) e Islebe et al. (2009). Los daños se clasificaron como ruptura de troncos (rotos a la mitad del tronco y rotos en la base del tronco), derribados, descopados (pérdida de ramas primarias y secundarias), inclinados (con y sin raíz expuesta), doblados y secos. El nivel de daño en cada sitio se designó como alto, medio o bajo; sin embargo, para toda el área y en concordancia con los demás análisis se usaron sólo las categorías de alto y bajo impacto. Adicionalmente, se anotó la producción de flores, frutos, hojas y semillas en el momento del muestreo, tomándose como referencia los sitios de bajo daño.

Relación hábitat-fauna. Con el listado de plantas obtenidas en el muestreo de vegetación se realizó una búsqueda en la literatura de las plantas que consumen los principales vertebrados neotropicales. Con el fin de asignar a las especies su importancia como fuente de alimento para los vertebrados del presente estudio, se realizaron 15 entrevistas informales y a profundidad a cazadores y conocedores de la fauna local del área de estudio. En las entrevistas se preguntó si la planta era consumida por la fauna, qué animales la consumen y qué parte de la planta aprovechan. 
Con estos datos se elaboró una lista de especies de importancia alimenticia y se determinó la calidad de hábitat en función del alimento potencial.

Análisis estadísticos. Se agruparon los sitios por intensidad de daño, para lo cual se separaron en alto y bajo impacto. Para verificar el ajuste normal de los datos se uso la prueba de Kolmogorov con el $p$-valor de Lilliefors corregido (Zar, 1996). Puesto que no se encontró distribución normal de los datos y homogeneidad de varianzas, aun con transformaciones, se procedió con análisis no paramétricos. Con los parámetros estructurales de la vegetación, los atributos del hábitat y las abundancias de fauna, se realizaron comparaciones en cada intensidad de daño utilizando pruebas de Mann-Whitney (Zar, 1996), para definir si existen diferencias en el hábitat que expliquen o ayuden a interpretar la calidad de hábitat y las abundancias de fauna.

Se calculó el índice de diversidad de Shannon ( $\left.\mathrm{H}^{\prime}\right)$ para determinar la diversidad basada en la proporción de abundancia de las especies vegetales y con la prueba $t$ de Hutcheson se comprobó la hipótesis de similitud o diferencia de la diversidad-abundancia en la condición de alto y bajo impacto (Magurran, 1988). Además, se estimó el índice de Simpson (D) y se realizaron comparaciones para determinar diferencias en la dominancia de las especies en condiciones de alto y bajo impacto (prueba $\mathrm{U}$ de Mann-Whitney; Magurran, 1988; Zar, 1996). Para diferenciar la composición de especies en los sitios de alto y bajo impacto, se determinaron los índices de Sorensen $\left(\mathrm{I}_{\mathrm{S}}\right)$, basado en datos cualitativos (presencia-ausencia) y el de Morisita-Horn $\left(\mathrm{I}_{\mathrm{MH}}\right)$, basado en datos cuantitativos de las especies (Magurran, 1988). Con éstos se comprobó la hipótesis de homogeneidad del hábitat en términos de la abundancia y composición de especies de árboles del área de estudio.

Se realizaron regresiones lineales entre la abundancia relativa de rastros de fauna y las posibles variables explicativas (e.g., porcentaje de daño en la vegetación, IVI), lo cual se usa regularmente para evaluar el grado de asociación debido a la influencia potencial del hábitat en la abundancia de fauna (Galetti et al., 2001; Arroyo-Rodríguez y Mandujano, 2006; Arroyo-Rodríguez et al., 2007). De igual manera se examinó la misma relación de las abundancias de fauna con el IVI, área basal y cobertura de las especies arbóreas registradas como fuente de alimento.

\section{Resultados}

Evaluación del hábitat. De acuerdo con el muestreo de vegetación, se registraron 3253 árboles y se identificaron 100 especies leñosas pertenecientes a 43 familias en un área de $20000 \mathrm{~m}^{2}$ (Apéndice). Las familias con la mayor densidad de individuos y que concentran casi el $50 \%$ de las especies presentes fueron Euphorbiaceae (17.3\%), Sapotaceae (15.2\%), y Fabaceae (13.2\%). En términos de diversidad arbórea no se encontró diferencia significativa entre los sitios de alto $\left(\mathrm{H}^{\prime}=2.86 \pm 0.36\right)$ y bajo impacto $\left(\mathrm{H}^{\prime}=\right.$ $2.68 \pm 0.27 ; \mathrm{t}=0.21 ;$ g.l. $=1541.5 ; p>0.05)$. De la misma forma, en términos de dominancia de las especies medido con el índice de Simpson, no se observaron diferencias ( $p>$ $0.05)$ en los sitios de alto impacto $(14.28 \pm 6.9)$ con respecto a los de bajo impacto (12.98 \pm 7.7 ; Cuadro 1). Asimismo, se encontró gran similitud en las condiciones de alto y bajo impacto tanto en la presencia-ausencia de especies $\left(\mathrm{I}_{\mathrm{S}}=\right.$ $84 \%)$ como en la abundancia de especies $\left(\mathrm{I}_{\mathrm{MH}}=83 \%\right)$. En cuanto a los parámetros del hábitat (cobertura, área basal, volumen y densidad total) no se encontraron diferencias de acuerdo con la intensidad de daño del huracán; tampoco se observó diferencia en estos parámetros con las especies de uso alimenticio (Cuadro 1).

Evaluación del daño. El daño en la vegetación fue la única variable que presentó diferencias en el hábitat cuando se evaluó la intensidad de impacto. La zona de alto impacto presentó mayor porcentaje de árboles dañados que la de bajo impacto ( $\mathrm{U}=154 ; p=0.0002$; Cuadro 1$)$. El mayor daño en la vegetación se observó entre 35 y $60 \mathrm{~km}$ de distancia perpendicular al ojo del huracán y los efectos disminuyeron gradualmente hasta $120 \mathrm{~km}$ de distancia (de 1 a 15\% de individuos arbóreos dañados; Fig. 2). Entre las categorías de daño se encontraron árboles con ruptura a nivel del tronco (40\%), descopados (32\%), inclinados $(11 \%)$, doblados $(7 \%)$, secos $(6 \%)$ y derribados $(3 \%)$.

De un total de 1560 árboles medidos en el área de alto impacto, el 98\% de los individuos presentaba hojas nuevas; sin embargo, solamente el 3\% de los árboles se encontró con flor, fruto y semilla. En el área de bajo impacto, el 83\% de los árboles $(\mathrm{n}=1392)$ presentaba hojas y sólo se encontró el $3 \%$ de individuos con fruto y el $1 \%$ con flor.

Especies de importancia alimenticia. Del total de especies arbóreas registradas en el área de estudio, alrededor del $65 \%$ fueron reconocidas, por informantes clave, con alguna importancia como alimento para herbívoros (frugívoros, ramoneadores y folívoros) y para varios omnívoros que incluyen en su dieta plantas o partes de ellas (hojas, flores o frutos). Asimismo, en toda el área de muestreo, el porcentaje promedio de especies de árboles que la fauna consume como alimento fue del $77 \%$ de los individuos presentes. En su conjunto, estas mismas especies reúnen el $74 \%$ del valor de importancia de los árboles presentes, el $70 \%$ de la densidad y el $76 \%$ de la cobertura, área basal y volumen total. Cuando se realizaron las comparaciones de estos porcentajes entre los sitios de alto y bajo impacto por el huracán no se encontraron diferencias significativas (Cuadro 1).

Las especies arbóreas con uso alimenticio para la fauna que presentaron el mayor valor de importancia (IVI, entre 
Cuadro 1. Valores promedio de los atributos y parámetros del hábitat $( \pm \mathrm{DS})$ del área de estudio ( $\mathrm{n}=20$ parcelas) de acuerdo con la intensidad de daño

Alto impacto

Atributos del hábitat

Riqueza (número de sp.)

H'

E

D
28.8 (4.92)

$2.86(0.36)$

$0.85(0.08)$

$14.28(6.98)$
Bajo impacto

$27.1(2.42)$

$2.68(0.27)$

$0.81(0.07)$

$12.98(7.74)$ $p$

0.22

0.36 *

0.28

0.65

Parámetros del hábitat

Cobertura (m2)

Área basal (m2)

Volumen (m2)

Densidad (ind/ha)

\% Árboles dañados

$$
\begin{gathered}
2023.97(306.89) \\
321.37(69.98) \\
221.6(39.22) \\
167.3(41.97) \\
37.32(14.29)
\end{gathered}
$$

78.63 (6.18)

79.01 (7.62)

$76.18(9.45)$

80.27 (5.88)

$\begin{array}{cc}1959.66(196.93) & 0.7 \\ 305.3(55.76) & 0.87 \\ 199.64(21.06) & 0.15 \\ 157.9(23.88) & 0.76\end{array}$

$5.54(6.07)$

0.0002

Especies arbóreas/alimento

$\begin{array}{lccr}\text { \% IVI } & 78.63(6.18) & 72.46(10.08) & 0.08 \\ \text { \% Cobertura } & 79.01(7.62) & 73.77(11.14) & 0.25 \\ \text { \% Área basal } & 76.18(9.45) & 75.35(14.21) & 0.82 \\ \text { \% Densidad } & 80.27(5.88) & 73.96(9.74) & 0.11\end{array}$

H', índice de diversidad de Shannon; E, equitabilidad; D, diversidad de Simpson. Significancia $p<0.05$ (prueba t de Hutcheson* y U de Mann-Whitney).

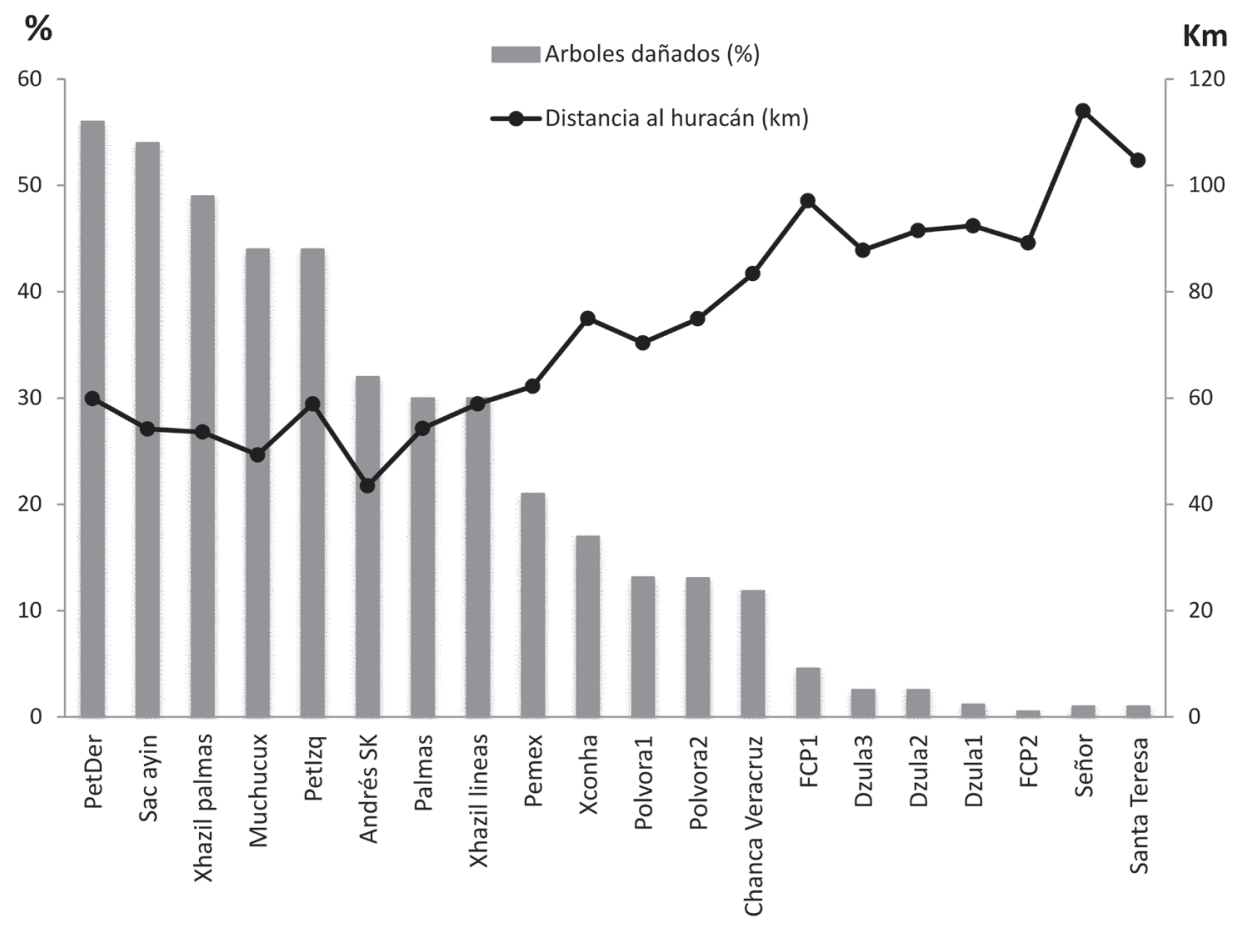

Figura 2. Sitios de muestreo con su respectivo porcentaje de daño y distancia al ojo del huracán en kilómetros. 
Cuadro 2. Abundancia relativa promedio de fauna ( $\pm \mathrm{DS})$ en los sitios con alto y bajo impacto del huracán (\# rastros/km)

\begin{tabular}{lcccc}
\hline Grupo funcional $^{*}$ & Alto & Bajo & $U$ & $p$ \\
\hline Herbívoros (Total) & $0.72(0.46)$ & $1.06(0.5)$ & -6.4 & $<0.0001$ \\
Frugívoros & $0.26(0.26)$ & $0.45(0.34)$ & -6.08 & $<0.0001$ \\
Frugívoro-ramoneadores & $0.45(0.34)$ & $0.58(0.36)$ & -3.97 & 0.0001 \\
Frugívoro-omnívoros & $0.22(0.23)$ & $0.24(0.24)$ & -0.5 & 0.6161 \\
Omnívoro-frugívoros & $0.58(0.29)$ & $0.46(0.26)$ & 4.3 & $<0.0001$ \\
Omnívoro & $1.26(0.58)$ & $1.09(0.49)$ & 3.12 & 0.0018 \\
\hline
\end{tabular}

* En función de la dieta. U, prueba de Mann-Whitney. Significancia $p<0.05$.

Cuadro 3. Relación lineal entre el porcentaje de daño en el hábitat y la abundancia relativa de rastros (rastros/km) por grupos de fauna. $\mathrm{R}^{2}=$ variabilidad explicada por el modelo en \%; $\mathrm{r}=$ correlación de Pearson que indica la dirección y fuerza de la relación entre la variable dependiente y la explicativa. $p<0.05$

\begin{tabular}{lccccc}
\hline Variable dependiente vs. Daño & $F$ & $p$ & $R^{2}(\%)$ & $r$ & $p$ \\
\hline Herbívoros (Total) & 24.7 & $\mathbf{0 . 0 0 0 1}$ & $\mathbf{5 7 . 9}$ & $\mathbf{- 0 . 7 6}$ & $<\mathbf{0 . 0 5}$ \\
Frugívoros & 6.7 & $\mathbf{0 . 0 1 8 6}$ & $\mathbf{2 7 . 1}$ & $\mathbf{- 0 . 5 2}$ & $<\mathbf{0 . 0 5}$ \\
Frugívoro-ramoneadores & 8.5 & $\mathbf{0 . 0 0 9 1}$ & $\mathbf{3 2 . 2}$ & $\mathbf{- 0 . 5 7}$ & $<\mathbf{0 . 0 5}$ \\
Frugívoro-omnívoros & 0.65 & 0.43 & 3.5 & -0.19 & 0.43 \\
Omnívoro- frugívoros & 1.2678 & 0.27 & 6.6 & 0.25 & 0.27 \\
Omnívoro & 1.3951 & 0.2529 & 7.19 & 0.2682 & 0.2529 \\
\hline
\end{tabular}

paréntesis) fueron: yaiti Gymmanthes lucida (26.46), chackya Pouteria reticulata (17.91), chicozapote Manilkara zapota (15.16), chaca roja Bursera simaruba (13.48), boob Coccoloba spicata (8.71), tadzi Neea choriophylla (8.19), guayabillo Eugenia laevis (7.95), tzilil Diospyros cuneata (7.45) ramón Brosimum alicastrum (6.62), yaaxnik Vitex gaumeri (6.09), chechem Metopium brownei (5.55), ekulub Drypetes lateriflora (5.54), sac chaca Dendropanax arboreus (5.25), tastab Guettarda combsii (4.94), amapola Pseudobombax ellipticum (4.86) y chichboob Coccoloba cozumelensis (4.53), entre otras (Apéndice).

Abundancia de fauna por grupos funcionales. En general, los herbívoros (e.g., frugívoros y frugívoro-ramoneadores) presentaron significativamente menores abundancias relativas promedio en los sitios con alto impacto del huracán $(\mathrm{U}=-6.4 ; p<0.05)$; por el contrario, las especies frugívoroomnívoros no presentaron cambio de abundancia, y los omnívoro-frugívoros y el más omnívoro presentaron significativamente mayores abundancias en los sitios con alto impacto $(\mathrm{U}=4.3 ; p<0.05$ y $\mathrm{U}=3.12 ; p<0.05$, respectivamente; Cuadro 2).

Relación hábitat-abundancias de fauna. En general, el daño en la vegetación presentó un efecto negativo en la abundancia relativa de herbívoros $(\mathrm{F}=24.72 ; \mathrm{r}=-0.76 ; p<$ 0.0001 ). Dentro de este grupo, tanto los frugívoros (i.e., cereque, tepezcuintle; $\mathrm{F}=6.7 ; \mathrm{r}=-0.52 ; p<0.01)$ como los frugívoro-ramoneadores (i.e., venado cola blanca y temazate; $\mathrm{F}=8.5 ; \mathrm{r}=-0.57 ; p<0.009)$ mostraron significativamente menores abundancias relativas en relación con el daño en la vegetación. Por su parte, el grupo de especies que posee algún grado de omnivoría en su dieta (i.e., hocofaisán, pavo ocelado, coatí, pecarí de collar y armadillo) no presentó una respuesta significativa en relación con el daño a los árboles del área de estudio (Cuadro 3).

\section{Discusión}

Evaluación del hábitat. En términos generales, a un año de evaluación del huracán, se encontró homogeneidad en la riqueza, equitabilidad y proporción de abundancia de las especies arbóreas en la condición de alto y bajo impacto, aspecto importante para la evaluación de la abundancia relativa de fauna en un hábitat considerablemente continuo, donde la principal variación en el hábitat estuvo determinada por los daños cualitativos en la vegetación arbórea. Sin embargo, esto no implica que no existan cambios en árboles de diámetros menores o en otras formas de vida (herbáceas y arbustos), lo cual no fue evaluado en el presente estudio.

El área basal, cobertura, volumen y densidad de especies no mostraron diferencias en la condición de alto y bajo impacto. Sánchez e Islebe (1999) registraron disminución en la densidad, área basal, cobertura y diversidad de árboles después del huracán Gilberto (1988); la diferencia radicó 
en que estos autores contaban con evaluaciones previas al disturbio y lograron evaluar el cambio inmediatamente después del huracán en sus parcelas de estudio. Lo anterior evidencia la importancia de contar con una referencia del sitio antes de la perturbación y con registros en los primeros meses post-disturbio. No obstante, a pesar de que no se detectaron los efectos negativos en la estructura de la vegetación de manera cuantitativa, se identificó el proceso de recuperación estructural a un año del paso del huracán; lo cual muestra que el crecimiento de ramas, rebrotes y producción de hojas corresponde con una alta resiliencia del sistema ante perturbaciones naturales. Calderón-Aguilera et al. (2011) citaron una severidad mediana y una tasa de recuperación rápida de la selva ante los huracanes, en comparación con los incendios naturales con severidad mayor y lenta recuperación. Por ejemplo, Islebe et al. (2009) registraron defoliación total en parcelas de selva mediana subperennifolia después del huracán Dean y un mes más tarde, la recuperación del $80 \%$ del follaje. De hecho, tanto los atributos como lo parámetros estructurales del hábitat, fueron ligeramente mayores en el área de alto impacto, debido al proceso de recuperación del hábitat.

Evaluación del daño. La evaluación del daño en el hábitat se realizó a partir de $40 \mathrm{~km}$ perpendiculares a la ruta del ojo del huracán, donde la afectación máxima mostró daños en el 56\% de los árboles medidos. En una evaluación rápida, Matyas et al. (2007) encontraron que los mayores daños en la vegetación ocurrieron en una franja de aproximadamente $50 \mathrm{~km}(25 \mathrm{~km}$ a cada lado de la ruta del ojo del huracán Dean), con 40 a $70 \%$ de daño en los árboles, lo cual coincide con los daños encontrados por Islebe et al. (2009) y en el presente estudio. Desde el presente enfoque de estudio, el efecto del huracán en el hábitat, en términos cualitativos, fue más evidente en los daños a los árboles. Entre las categorías de daño, las que representan mayores efectos sobre la productividad de los árboles, son la condición de ruptura de troncos y la pérdida excesiva de ramas, principalmente por el elevado porcentaje de individuos y por la dificultad que representa la recuperación de ramas nuevas, así como la sincronización fenológica de hojas, flores y frutos.

El periodo de muestreo en la vegetación corresponde con la fase caducifolia de la selva mediana subperennifolia y teóricamente con la mayor producción de flores y parte de la producción de frutos (Miranda, 1958; PorterBolland et al., 2009; Niembro et al., 2010); no obstante, no se encontró este patrón caducifolio ni fenológico, lo cual expresa la resiliencia de la vegetación ante el estrés producido por el disturbio. La escasa producción de flores y frutos observada durante el periodo de estudio (2 años y medio después del huracán) muestra que el efecto de estrés post-huracán en la fenología puede cubrir una escala temporal y espacial mayor a la estudiada.
La elevada producción de hojas de manera anómala en el periodo caducifolio de la selva en toda el área de estudio, pero en particular en la zona de mayor daño, puede ser una respuesta para compensar las condiciones de estrés provocadas por el daño estructural. Por otra parte, la mayor cantidad de árboles sin hojas en los sitios con bajo impacto muestra un patrón fenológico más común, de acuerdo con el aspecto caducifolio de la vegetación (Miranda, 1958). Es posible que persista un efecto negativo de mayor escala no detectado en la fenología de las especies, a pesar de ser evidente la disminución del daño físico en los árboles de esta área. Para el caso de una selva tropical seca, Imbert y Portecop (2008) mencionan que la recuperación después de un huracán puede ocurrir en el transcurso de 2 a 6 años, lo cual incluye producción de hojarasca, crecimiento de ramas, reclutamiento de individuos e inicio de la sincronización fenológica de los árboles. Sin embargo, la recuperación total de las fenofases, así como de la estructura, composición, densidad y área basal de los árboles al estado pre-disturbio, puede tomar un periodo de tiempo de 10 a 30 años (Weaver, 1986; Crow, 1980; Imbert y Portecop, 2008).

Relación entre el daño del hábitat con las abundancias relativas de fauna. Existen predicciones generales que establecen que la omnivoría, comparada con la herbivoría, es una característica que permite a las especies soportar mejor una perturbación (McKinney y Lockwood, 1999; Vázquez y Simberloff, 2002; Tejeda-Cruz y Sutherland, 2004). Aunque el estado de estrés se presenta para todas las especies animales, sólo fue significativa para fauna con algún grado de herbivoría (frugívoros y frugívoros-folívoros). Esta misma respuesta fue confirmada mediante un enfoque pre y post-huracán Dean en un ejido forestal de la zona maya, donde se encontró una fuerte disminución de abundancias de especies frugívoras y ramoneadoras después del disturbio (Ramírez-Barajas et al., 2012). Esto significa que el daño en el hábitat tiene una fuerte repercusión en la disminución significativa de la abundancia de especies cuya dieta se basa en frutos y hojas. Por lo tanto, el daño en el hábitat resultó ser una variable que en buena medida explica el comportamiento de las abundancias relativas de estas especies en el área de estudio. El grado de frugivoría o herbivoría es una característica altamente sensible a la perturbación, debido a la alta probabilidad de escasez de frutos, hojas y flores en un hábitat fragmentado o alterado después de un disturbio (Gray et al., 2007; Kikira et al., 2008; Vetter et al., 2011; Bonnell et al., 2011). Los eventos de disturbio, en particular los huracanes, son identificados porque sus efectos negativos sobre especies frugívoras son mayores que en insectívoras y omnívoras (Lynch, 1991; Waide, 1991; Will, 1991; Wunderle et al., 1992; Pavelka et al., 2003; Tejeda-Cruz y Sutherland, 2004). 
La relación del daño en el hábitat no fue tan clara para los frugívoro-omnívoros (pavo ocelado y hocofaisán). Sin embargo, los omnívoros-frugívoros (pecarí de collar, coatí) y el omnívoro (armadillo) presentaron una tendencia a incrementar sus abundancias en las zonas de mayor daño, lo cual puede interpretarse como una mayor resistencia al estrés, al recurrir a otros alimentos diferentes de las plantas. Esta misma respuesta pudo registrarse con datos pre y post-huracán en el ejido Petcacab, donde se observa una ventaja relativa del carácter omnívoro y generalista de estas mismas especies (Ramírez-Barajas et al. 2012). Un mayor grado de omnivoría (en el sentido de una menor dependencia de las plantas), introduce un mecanismo de amortiguamiento potencial, el cual puede disminuir la severidad y duración de los cambios inducidos por la perturbación. Además, es una estrategia de vida oportunista para especies adaptadas a soportar condiciones limitadas de alimento en las selvas (Bodmer, 1989; Fagan, 1997). En efecto, los omnívoros tienden a ser oportunistas e incluyen en sus dietas raíces, insectos y otros invertebrados del suelo que pueden compensar la carencia de frutos, flores y hojas.

El daño en el hábitat fue la variable con mayor influencia en la abundancia relativa de fauna en el área de estudio. Otras variables como la diversidad y los atributos de la vegetación no presentaron una relación significativa con las abundancias de fauna. Esto puede estar relacionado con el efecto del diseño de muestreo en la vegetación, ya que éste cubrió una fase del proceso de recuperación post-huracán de la vegetación y no se obtuvieron datos comparativos como referencia pre-disturbio. Sin embargo, se encontró una respuesta diferencial por grupos funcionales de fauna, donde los herbívoros presentaron un efecto negativo en sus abundancias relativas de rastros en relación con el daño en la vegetación, mientras que la característica omnívora de los grupos permitió soportar el estrés post-huracán e incluso aprovechar las condiciones de la perturbación. Los resultados obtenidos revelan la necesidad de contar con estudios a largo plazo para evaluar tanto el impacto inmediato de los huracanes sobre la fauna silvestre y la vegetación como el proceso de recuperación del hábitat y especies. Comprender la resiliencia de los ecosistemas y especies es sin duda un tema prioritario ante los actuales escenarios de cambio global, para los que se prevén disturbios naturales con mayor frecuencia y de gran magnitud.

\section{Agradecimientos}

A la población y autoridades de los ejidos Petcacab, Andrés Quintana Roo, Kopchen, Dzula, X-Hazil, X-Conha, Felipe Carrillo Puerto e X-Maben (Señor), y a las autoridades de la Reserva de la Biosfera Sian Ka'an, por permitir el trabajo de investigación. A Sophie Calmé, por todo su apoyo en innumerables partes del proyecto; a Héctor Hernández y Salvador Mandujano, por las ideas y recomendaciones; a los revisores anónimos por sus recomendaciones para el mejoramiento del manuscrito. De manera especial, a Alejandro Tuz y a Margarito, quienes apoyaron durante todo el trabajo de campo y monitoreo. A doña Mila, Carmen, Rigoberto Váldez y Ángela Chan, por recibirnos en sus hogares. A Malú, por su gran ayuda en la captura de datos. A Holger Weissenberger, por la elaboración de diversos mapas. A Julio Moure, por su apoyo para el financiamiento PDUD-COMPACT a través de SEYBA. El presente proyecto no hubiera sido posible sin los fondos económicos de SPFEQROO, CONACYT, CONAFORPROCYMAF II, GEF COMPACT-Sian Ka'an, ECOSUR y COQCYT, junto con la beca de doctorado para Pablo Jesús Ramírez Barajas, CONACYT-103627.

\section{Literatura citada}

Arriaga, L., J. M. Espinoza, C. Aguilar, E. Martínez, L. Gómez y E. Loa. 2000. Regiones terrestres prioritarias de México. Escala de trabajo 1: 1000 000. rtp_149(1).pdf. CONABIO, México, D. F. http://www.conabio.gob.mx/conocimiento/ regionalizacion/doctos/Tsureste.html; última consulta: 8.XII.2012.

Aranda, M. 2000. Huellas y otros rastros de los mamíferos grandes y medianos de México. CONABIO/ Instituto de Ecología, Xalapa, Veracruz. 212 p.

Arroyo-Rodríguez, V. y S. Mandujano. 2006. Forest fragmentation modifies habitat quality for Alouatta palliata. International Journal of Primatology 27:1079-1096.

Arroyo-Rodríguez, V., S. Mandujano, J. Benítez-Malvido y C. Cuende-Fanton. 2007. The influence of large tree density on howler monkey (Alouatta palliata mexicana) presence in very small rainforest fragments. Biotropica 39:760-766.

Bodmer, R. E. 1989. Ungulate biomass in relation to feeding strategy within Amazonian forests. Oecologia 81:547-50.

Bonnell, T. R., R. Reyna-Hurtado y C. A. Chapman. 2011. Postlogging recovery time is longer than expected in an East African tropical forest. Forest Ecology and Management 261:855-864.

Burnham, K. P., D. R. Anderson y J. L. Laake. 1980. Estimation of density from line transect sampling of biological populations. Wildlife Monographs 72:1-202.

Calderón-Aguilera, L. E., V. H. Rivera-Monroy, L. Porter-Bolland, A. Martínez-Yrízar, L. B. Ladah, M. Martínez-Ramos, J. Alcocer, A. L. Santiago-Pérez, H. A. Hernández-Arana, V. M. Reyes-Gómez, D. R. Pérez-Salicrup, V. Díaz-Nuñez, J. Sosa-Ramírez, J. Herrera-Silveira y A. Búrquez. 2011. An assessment of natural and human disturbance effects on Mexican ecosystems: current trends and research gaps. 
Biodiversity and Conservation DOI: 10.1007/s10531-0110218-6.

Carrillo, E., G. Wong y A. D. Cuarón. 2000. Monitoring mammal populations in Costa Rican protected areas under different hunting restrictions. Conservation Biology 14:1580-1591.

Chapman, C. A., L. J. Chapman, R. Wangham, K. Hunt, D. Gebo y L. Gardner. 1992. Estimators of fruit abundance of tropical trees. Biotropica 24:527-531.

Chapman, C. A., R. Wrangham y L. J. Chapman. 1994. Indices of habitat-wide fruit abundance in tropical forest. Biotropica 26:160-171.

Chiarello, A. G. 2000. Density population size of mammals in remnants of Brazilian Atlantic forest. Conservation Biology 14:1649-1657.

CONAGUA. 2007. Reseña del huracán Dean del océano Atlántico. http://smn.cna.gob.mx/ciclones/tempo2007/ atlantico/dean.pdf; última consulta: 13.III.2012

Crow, T. R. 1980. A rainforest chronicle: a 30-year record of change in structure and composition at El Verde, Puerto Rico. Biotropica 12:42-55.

Fagan, W. F. 1997. Omnivory as a stabilizing feature of natural communities. The American Naturalist 150:554-567.

Felton, A. M., L. M. Engstram, A. Felton y C. D. Knott. 2003. Orangutan population density, forest structure and fruit availability in handlogged and unlogged peat swamp forests in West Kalimantan, Indonesia. Biological Conservation 114:91-101.

Fischer, J y D. B. Lindenmayer. 2007. Landscape modification and habitat fragmentation: a synthesis. Global Ecology and Biogeography 16:265-280.

Fleming, T. H., R. Breitwisch y G. H. Whitesides. 1987. Patterns of tropical vertebrate frugivore diversity. Annual Review of Ecology and Systematics 18:91-109.

Galetti, M., A. Keuroghlian, L. Hanada y M. Inez-Morato. 2001. Frugivory and Seed Dispersal by the Lowland Tapir (Tapirus terrestris) in Southeast Brazil. Biotropica 33:723-726.

Gentry, A. H. 1982. Patterns of Neotropical plant species diversity. Evolutionary Biology 15:1-85.

Gray, M. A., S. L. Baldauf, P. J. Mayhew y J. K. Hill. 2007. The response of avian feeding guilds to tropical forest disturbance. Conservation Biology 21:133-141.

Hazell, D., J. M. Hero, D. Lindenmayer y R. Cunningham. 2004. A comparison of constructed and natural habitat for frog conservation in an Australian agricultural landscape. Biological Conservation 119:61-71.

Imbert, D. y J. Portecop. 2008. Hurricane disturbance and forest resilience: assessing structural vs. functional changes in a Caribbean dry forest. Forest Ecology and Management 255:3494-3501.

Islebe, G. A, N. Torrescano-Valle, M. Valdez-Hernández, M. TuzNovelo y H. Weissenberger. 2009. Efectos del impacto del huracán Dean en la vegetación del sureste de Quintana Roo,
México. Foresta Veracruzana 11:1405-7247.

Kent, M. y P. Coker. 1994. Vegetation description and analysis: a practical approach. John Wiley and Sons. Chichester. 363 p.

Kikira, J. M., N. Farwing y K. Bönhing-Gaese. 2008. Effects of local disturbance of tropical forests on frugivores and seed removal of a small-seeded Afrotropical tree. Conservation Biology 22:318-328.

Krebs, C. J. 2006. Mammals. In Ecological census techniques: a handbook. W. J. Sutherland. (ed.). Cambridge University Press, Cambridge. p. 351-410.

Kreuzer, M. P. y N. J. Huntly. 2003. Habitat-specific demography: evidence for source-sink population structure in a mammal, the pika. Oecologia 134:343-349.

Lynch, F. J. 1991. Effects of hurricane Gilbert on a dry tropical forest in the Yucatán Peninsula. Biotropica 23:488-496.

Lyra-Jorge, M. C., G. Ciocheti, V. R. Pivello y S. T. Meirelles. 2008. Comparing methods for sampling large-and mediumsized mammals: camera traps and track plots. European Journal of Wildlife Research 54:739-744.

McKinney, M. L. y J. L., Lockwood. 1999. Biotic homogenization: a few winners replacing many losers in the next mass extinction. Trends in Ecology and Evolution 14:450-453.

Magurran, A. E. 1988. Ecological diversity and its measurement. Princeton University Press, New Jersey. 179 p.

Matyas, C. J., L. Fernández-Salvador y S. Calmé. 2007. Tree damage in Quintana Roo, Mexico caused by hurricane Dean (2007). 28th Conference on Hurricanes and Tropical Meteorology. University of Florida, Gainsville. http://ams. confex.com/ams/28Hurricanes/techprogram/paper_137940. htm; última consulta: 20.V.2009.

Milton, K. 2008. Macronutrient patterns of 19 species of Panamanian fruits from Barro Colorado Island. Neotropical Primates 15:1-7.

Miranda, F. 1958. Estudios acerca de la vegetación. In Los recursos naturales del sureste y su aprovechamiento, tomo II, E. Beltrán (ed.). Instituto Mexicano de Recursos Naturales Renovables México, México, D. F. p. 215-271.

Mortelliti, A., G. Amori y L. Boitani. 2010. The role of habitat quality in fragmented landscapes: a conceptual overview and prospectus for future research. Oecologia 163:535-547.

Murie, O. J. y M. Elbroch. 2005. A field guide of animal tracks. Peterson Field Guide Series. Houghton Mifflin, Boston, Massachusetts. 391 p.

National Ocean and Atmosphere Administration (NOAA). 2011. Hurricane Gilbert - September 14-21, 1988. http://www. hpc.ncep.noaa.gov/tropical/rain/gilbert1988.html; última consulta: 13.III.2012.

Niembro, R. A., M. Vázquez-Torres y O. Sánchez-Sánchez. 2010. Árboles de Veracruz. Cien especies para la reforestación estratégica. Gobierno del Estado de Veracruz/ CEVCBINCRM/ Universidad Veracruzana, Xalapa. 253 p. Orellana, L. R., C. Espadas, C. Conde y C. García. 2009. Atlas. 
Escenarios de cambio climático en la península de Yucatán. Centro de Investigación Científica de Yucatán, Mérida, 111 p.

Pavelka, M. S., O. T. Brusselers, D. Nowak y A. M. Behie. 2003. Population reduction and social disorganisation in Alouatta pigra following a hurricane. International Journal of Primatology 24:1037-55.

Pickett, S. T. A. y P. S. White. 1985. The ecology of natural disturbance and patch dynamics. Academic, San Diego, California. 472 p.

Porter-Bolland, L., M. E. Medina-Abreo, J. Montoy-Koh, P. Montoy-Koh, G. Martín-Ek y G. May-Pacheco. 2009. Flora melífera de la montaña, Campeche: su importancia para la apicultura y la vida diaria. Comisión Nacional para el Conocimiento y Uso de la Biodiversidad/ Instituto de Ecología, Xalapa, Veracruz. 322 p.

Ramírez-Barajas, P. J., G. A. Islebe y S. Calmé. 2012. Impact of Hurricane Dean (2007) on game species of the Selva Maya. Biotropica 44:402-411.

Roberts, N. J. 2011. Investigation into survey techniques of large mammals: surveyor competence and camera-trapping vs. transect-sampling. Bioscience Horizons 4:40-49.

Sánchez, S. O. y G. Islebe. 1999. Hurricane Gilbert and structural changes in a tropical forest in south-eastern Mexico. Global Ecology and Biogeogeography 8:29-38.

Silveira, L., A. T. A. Jácomo y J. A. F. Diniz-Filho. 2003. Camera trap, line transect census and track surveys: a comparative evaluation. Biological Conservation 114:351-355.

Tanner, E. V. J., V. Kapos y J. R. Healey. 1991. Hurricane effects on forest ecosystems in the Caribbean. Biotropica 23:513-521.

Tejeda-Cruz, C. y W. J. Sutherland. 2004. Bird responses to shade coffee production. Animal Conservation 7:169-179.

Urquiza-Haas, T., C. A. Peres y P. M. Dolman. 2011. Large vertebrate responses to forest cover and hunting pressure in communal landholdings and protected areas of the Yucatan, Peninsula, Mexico. Animal Conservation 14:271-282.

van Horne, B. 1983. Density as a misleading indicator of habitat quality. Journal of Wildlife Management 47:893-901.

Vázquez, D. P. y D. Simberloff. 2002. Ecological specialization and susceptibility to disturbance: conjectures and refutations. The American Naturalist 159:606-623.

Vetter, D., M. M. Hansbauer, Z. Végvári y I. Storch. 2011. Predictors of forest fragmentation sensitivity in Neotropical vertebrates: a quantitative review. Ecography 34:1-8.

Waide, R. B. 1991. The effect of hurricane Hugo on bird populations in the Luquillo Experimental Forest, Puerto Rico. Biotropica 23:475-480.

Weaver, P. L. 1986. Hurricane damage and recovery in the montane forests of the Luquillo Mountains of Puerto Rico. Caribbean Journal of Science 22:53-70.

Will, T. 1991. Birds of a severely hurricane-damaged Atlantic coast rain forest in Nicaragua. Biotropica 23:497-507.

Wilson, G. J. y R. J. Delahay. 2001. A review of methods to estimate the abundance of terrestrial carnivores using field signs and observation. Wildlife Research 28:151-164.

Worman, C. O. y C. A. Chapman. 2006. Densities of two frugivorous primates with respect to forest and fragment tree species composition and fruit availability. International Journal of Primatology 27:203-225.

Wunderle, M. Jr., D. J. Lodge y R. B. Waide. 1992. Short-term effects of hurricane Gilbert on terrestrial bird populations on Jamaica. The Auk 109:48-166.

Zar, J. H. 1996. Biostatistical analysis. Prentice-Hall, Upper Saddle River, New Jersey. 960 p.

Zimmerman, J. K., M. R. Willig, L. R. Walker y W. L. Silver. 1996. Introduction: disturbance and Caribbean ecosystems. Biotropica 28:414-423.

Apéndice. Especies arbóreas en el área de estudio. N, número de individuos totales; IVI, índice de valor de importancia, ordenadas de mayor a menor valor; las 4 primeras representan aproximadamente el $73 \%$ del total de especies. Alimento, especies registradas como alimento para la fauna.

\begin{tabular}{llccccc}
\hline$\#$ & Nombre común & Especie & Familia & $N$ & IVI & Alimento \\
1 & Yaiti & Gymnanthes lucida & Euphorbiaceae & 414 & 26.46 & Sí \\
2 & Chackya & Pouteria reticulata & Sapotaceae & 234 & 17.91 & Sí \\
3 & Zapote & Manilkara zapota & Sapotaceae & 185 & 15.16 & Sí \\
4 & Chaca roja & Bursera simaruba & Burseraceae & 190 & 13.48 & Sí \\
5 & Boob & Coccoloba spicata & Polygonaceae & 114 & 8.71 & Sí \\
6 & Tadzi & Neea choriophylla & Nyctaginaceae & 102 & 8.19 & Sí \\
7 & Guayabillo & Eugenia laevis & Myrtaceae & 88 & 7.95 & Sí \\
8 & Tzilil & Diospyros cuneata & Ebenaceae & 85 & 7.45 & Sí \\
9 & Xul & Lonchocarpus xuul & Fabaceae & 90 & 6.99 & No \\
10 & Jabin & Piscidia piscipula & Fabaceae & 75 & 6.83 & No
\end{tabular}


Apéndice. Continúa.

\begin{tabular}{|c|c|c|c|c|c|c|}
\hline \# & Nombre común & Especie & Familia & $N$ & $I V I$ & Alimento \\
\hline 11 & Tabaquillo & Alseis yucatanensis & Rubiaceae & 76 & 6.63 & No \\
\hline 12 & Ramón & Brosimum alicastrum & Moraceae & 59 & 6.62 & Sí \\
\hline 13 & Kitamche & Caesalpinia gaumeri & Fabaceae & 60 & 6.20 & No \\
\hline 14 & Yaaxnik & Vitex gaumeri & Verbenaceae & 55 & 6.09 & Sí \\
\hline 15 & Canchunub & Thouinia paucidentata & Sapindaceae & 72 & 6.05 & No \\
\hline 16 & Tzalam & Lysiloma latisiliquum & Fabaceae & 54 & 5.71 & No \\
\hline 17 & Chechem & Metopium brownei & Anacardiaceae & 58 & 5.55 & Sí \\
\hline 18 & Ekulub & Drypetes lateriflora & Euphorbiaceae & 60 & 5.54 & Sí \\
\hline 19 & Sac chaca & Dendropanax arboreus & Araliaceae & 74 & 5.25 & Sí \\
\hline 20 & Tastab & Guettarda combsii & Rubiaceae & 61 & 4.94 & Sí \\
\hline 21 & Amapola & Pseudobombax ellipticum & Bombacaceae & 44 & 4.86 & Sí \\
\hline 22 & Chichboob & Coccoloba cozumelensis & Polygonaceae & 40 & 4.53 & Sí \\
\hline 23 & Perezcutz & Croton reflexifolius & Euphorbiaceae & 49 & 4.42 & Sí \\
\hline 24 & Elemuy & Malmea depressa & Annonaceae & 48 & 4.40 & Sí \\
\hline 25 & Tzol & Exothea paniculata & Sapindaceae & 41 & 4.35 & Sí \\
\hline 26 & Isinche & Casearia corymbosa & Euphorbiaceae & 40 & 4.32 & Sí \\
\hline 27 & Kaniste & Pouteria campechiana & Sapotaceae & 33 & 4.04 & Sí \\
\hline 28 & Kanasin & Lonchocarpus rugosus & Fabaceae & 34 & 4.01 & No \\
\hline 29 & Limonaria & Murraya paniculata & Rutaceae & 32 & 3.71 & No \\
\hline 30 & Laurelillo & Nectandra coriacea & Lauraceae & 38 & 3.54 & $\mathrm{Si}$ \\
\hline 31 & Katalox & Swartzia cubensis & Fabaceae & 27 & 3.46 & $\mathrm{Si}$ \\
\hline 32 & Huayuncox & Exothea diphylla & Sapindaceae & 26 & 3.10 & No \\
\hline 33 & Tzizilche & Gymnopodium floribundum & Polygonaceae & 23 & 2.93 & No \\
\hline 34 & Kaskat & Luehea speciosa o candida & Tiliaceae & 28 & 2.90 & $\mathrm{Si}$ \\
\hline 35 & Chaclolche & Erythrina standleyana & Fabaceae & 29 & 2.85 & No \\
\hline 36 & Pata vaca & Bauhinia divaricata & Fabaceae & 26 & 2.83 & No \\
\hline 37 & Chintok & Krugiodendron ferreum & Rhamnaceae & 25 & 2.68 & No \\
\hline 38 & Tres marías & Forchhammeria trifoliata & Capparidaceae & 22 & 2.63 & $\mathrm{Si}$ \\
\hline 39 & Huano botan & Sabal mexicana & Araceae & 19 & 2.60 & $\mathrm{Si}$ \\
\hline 40 & Sacyabin & Gliricidia sepium & Fabaceae & 22 & 2.58 & No \\
\hline 41 & Palo de rosa & Simira salvadorensis & Rubiaceae & 24 & 2.54 & $\mathrm{Si}$ \\
\hline 42 & Tziziya & Sideroxylon salicifolium & Sapotaceae & 21 & 2.17 & $\mathrm{Si}$ \\
\hline 43 & Huaya & Talisia olivaeformis & Sapindaceae & 15 & 2.06 & $\mathrm{Si}$ \\
\hline 44 & Tamay & Zuelania guidonia & Flacourtiaceae & 19 & 2.06 & No \\
\hline 45 & Negrito & Simarouba glauca & Simaroubaceae & 15 & 2.06 & $\mathrm{Si}$ \\
\hline 46 & Sacpa & Byrsonima bucidaefolia & Malpighiaceae & 14 & 2.02 & $\mathrm{Si}$ \\
\hline 47 & Cayumito & Chrysophyllum mexicanum & Sapotaceae & 18 & 1.85 & $\mathrm{Si}$ \\
\hline 48 & Copal & Protium copal & Burseraceae & 14 & 1.82 & $\mathrm{Si}$ \\
\hline 49 & Kekenche & Hyperbaena mexicana & Menispermaceae & 12 & 1.75 & $\mathrm{Si}$ \\
\hline 50 & Majahua & Hampea trilobata & Malvaceae & 15 & 1.73 & No \\
\hline 51 & Guayacan & Guaiacum sanctum & Zygophyllaceae & 12 & 1.40 & No \\
\hline
\end{tabular}


Apéndice. Continúa.

\begin{tabular}{|c|c|c|c|c|c|c|}
\hline \# & Nombre común & Especie & Familia & $N$ & $I V I$ & Alimento \\
\hline 52 & Kaax & Randia longiloba & Rubiaceae & 10 & 1.39 & $\mathrm{Si}$ \\
\hline 53 & Kishtel & Gyrocarpus jatrophifolius & Hernandiaceae & 10 & 1.37 & $\mathrm{Si}$ \\
\hline 54 & Hopchejon & Annona reticulata primigenia & Annonaceae & 13 & 1.31 & $\mathrm{Si}$ \\
\hline 55 & Tojyub & Coccoloba cardiophylla & Polygonaceae & 11 & 1.31 & $\mathrm{Si}$ \\
\hline 56 & Tankasche & Pilocarpus racemosus & Rutaceae & 19 & 1.24 & No \\
\hline 57 & Choy & Cochlospermum vitifolium & Cochlospermaceae & 8 & 1.18 & $\mathrm{Si}$ \\
\hline 58 & Chiit & Thrinax radiata & Arecaceae & 11 & 1.11 & $\mathrm{Si}$ \\
\hline 59 & Despeinada & Beaucarnea pliabilis & Nolinaceae & 8 & 1.06 & No \\
\hline 60 & Tusikche & sp. 1 & & 6 & 1.04 & No \\
\hline 61 & Belsininche & Alvaradoa amorphoides & Simaroubaceae & 6 & 1.02 & No \\
\hline 62 & Caoba & Swietenia macrophylla & Meliaceae & 5 & 1.01 & $\mathrm{Si}$ \\
\hline 63 & Caracolillo & Sideroxylon foetidissimum & Sapotaceae & 5 & 1.00 & $\mathrm{Si}$ \\
\hline 64 & Ceiba & Ceiba pentandra & Bombacaceae & 8 & 1.00 & $\mathrm{Si}$ \\
\hline 65 & Chilillo & Rourea glabra & Connaraceae & 6 & 0.97 & $\mathrm{Si}$ \\
\hline 66 & Napche & Ximenia americana & Olacaceae & 5 & 0.91 & $\mathrm{Si}$ \\
\hline 67 & Huano blanco & Sabal japa & Araceae & 10 & 0.85 & $\mathrm{Si}$ \\
\hline 68 & Uvillo & Coccoloba acapulcensis & Polygonaceae & 5 & 0.82 & $\mathrm{Si}$ \\
\hline 69 & Yaax ek & verde lucero & & 5 & 0.81 & No \\
\hline 70 & Siricote & Cordia dodecandra & Boraginaceae & 4 & 0.75 & $\mathrm{Si}$ \\
\hline 71 & Palo ruda & Diphysa carthagenensis & Fabaceae & 4 & 0.74 & $\mathrm{Si}$ \\
\hline 72 & Hupich & sp. 2 & & 4 & 0.73 & No \\
\hline 73 & Kulinche & Astronium graveolens & Anacardiaceae & 4 & 0.65 & No \\
\hline 74 & Higo & Ficus sp 1 & Moraceae & 4 & 0.59 & $\mathrm{Si}$ \\
\hline 75 & Akitz & Thevetia gaumeri & Apocynaceae & 3 & 0.45 & $\mathrm{Si}$ \\
\hline 76 & Chacte & Lonchocarpus guatemalensis & Fabaceae & 3 & 0.44 & No \\
\hline 77 & Kum & Crysophila argentea & Araceae & 6 & 0.41 & $\mathrm{Si}$ \\
\hline 78 & Flor de mayo & Plumeria obtusa & Apocynaceae & 2 & 0.40 & No \\
\hline 79 & Limoncillo & Jacquinia macrocarpa & Theophrastaceae & 3 & 0.39 & $\mathrm{Si}$ \\
\hline 80 & Ichbach & Allophylus cominia & Sapindaceae & 3 & 0.39 & $\mathrm{Si}$ \\
\hline 81 & Bojom & Cordia gerascanthus & Boraginaceae & 2 & 0.36 & $\mathrm{Si}$ \\
\hline 82 & Guayabillo hoja ancha & Eugenia sp. & Myrtaceae & 4 & 0.35 & $\mathrm{Si}$ \\
\hline 83 & Sacahua & Ficus sp. 2 & Moraceae & 2 & 0.34 & $\mathrm{Si}$ \\
\hline 84 & Jobillo & Spondias purpurea & Anacardiaceae & 2 & 0.34 & $\mathrm{Si}$ \\
\hline 85 & Piim & Ceiba schottii & Bombacaceae & 3 & 0.30 & No \\
\hline 86 & Yuy & Casimiroa tetrameria & Rutaceae & 3 & 0.29 & $\mathrm{Si}$ \\
\hline 87 & Granadillo & Platymiscium yucatanum & Fabaceae & 1 & 0.25 & No \\
\hline 88 & Jaboncillo & Sapindus saponaria & Sapindaceae & 2 & 0.21 & No \\
\hline 89 & Cacaoche & Zygia stevensonii & Fabaceae & 1 & 0.21 & $\mathrm{Si}$ \\
\hline 90 & Canela de cuyo & Canella winterana & Canellaceae & 1 & 0.21 & $\mathrm{Si}$ \\
\hline 91 & Puckte & Bucida buceras & Combretaceae & 1 & 0.21 & No \\
\hline 92 & Pomolche & Jatropha gaumeri & Euphorbiaceae & 1 & 0.21 & $\mathrm{Si}$ \\
\hline
\end{tabular}


Revista Mexicana de Biodiversidad 83: 1194-1207, 2012

DOI: $10.7550 / \mathrm{rmb} .27964$

Apéndice. Continúa.

\begin{tabular}{|c|c|c|c|c|c|c|}
\hline \# & Nombre común & Especie & Familia & $N$ & $I V I$ & Alimento \\
\hline 93 & Pacalche & Esenbeckia berlanderi & Rutaceae & 1 & 0.19 & $\mathrm{Si}$ \\
\hline 94 & Pimientillo & sp. 3 & & 1 & 0.19 & No \\
\hline 95 & Jobo & Spondias mombin & Anacardiaceae & 1 & 0.18 & $\mathrm{Si}$ \\
\hline 96 & Mora & Guazuma ulmifolia & Malvaceae & 1 & 0.18 & $\mathrm{Si}$ \\
\hline 97 & Payche & Achyranthes aspera & Amaranthaceae & 1 & 0.18 & No \\
\hline 98 & Chimay & Acacia milleriana & Fabaceae & 1 & 0.18 & No \\
\hline 99 & Chacteviga & Caesalpinia mollis & Fabaceae & 1 & 0.18 & No \\
\hline 100 & Katsim & Acacia riparia & Fabaceae & 1 & 0.17 & No \\
\hline
\end{tabular}

